\title{
Efecto de la relación área-volumen en la sinterización de varistores cerámicos basados en $\mathrm{ZnO}-\mathrm{Bi}_{2} \mathrm{O}_{3}$.
}

\author{
M. A. DE LA RUbiA, M. PEITEADO, J. F. FERNANDEZ, A. C. CABALlero \\ Departamento de Electrocerámica. Instituto de Cerámica y Vidrio, CSIC. 28500 Arganda del Rey, Madrid.
}

\begin{abstract}
La sinterización de varistores cerámicos basados en el sistema $\mathrm{ZnO}-\mathrm{Bi}_{2} \mathrm{O}_{3}-\mathrm{Sb}_{2} \mathrm{O}_{3}$ requiere temperaturas del orden de 1150 $1200^{\circ} \mathrm{C}$ y ocurre en presencia de una fase líquida rica en $\mathrm{Bi}_{2} \mathrm{O}_{3}$ que se forma entre 700 y $800^{\circ} \mathrm{C}$ dependiendo de la composición concreta del material. La volatilización incontrolada de $\mathrm{Bi}_{2} \mathrm{O}_{3}$ puede alterar notablemente la respuesta eléctrica del material, ya que este componente resulta crítico para la configuración de los bordes de grano activos. Por otro lado, la completa oxigenación del material durante la sinterización también resulta determinante en la respuesta eléctrica final del varistor. Consecuentemente, la relación área-volumen de los compactos en verde se convierte en un parámetro relevante durante la sinterización del material. En el presente trabajo se han estudiado las características microestructurales y la respuesta intensidad-voltaje de varistores cerámicos de idéntica composición, sinterizados a partir de compactos con diferente relación áreavolumen. Se ha observado que, dependiendo de la temperatura de sinterización, existe un valor de la relación área-volumen a partir del cual la respuesta eléctrica del material se degrada dramáticamente.
\end{abstract}

Palabras clave: varistor cerámico, relación área-volumen, sinterización, respuesta eléctrica.

Effect of the area-volume ratio in the sintering of $\mathrm{ZnO}-\mathrm{Bi}_{2} \mathrm{O}_{3}$ based ceramic varistors.

Sintering of ceramic varistors based on the system $\mathrm{ZnO}-\mathrm{Bi}_{2} \mathrm{O}_{3}-\mathrm{Sb}_{2} \mathrm{O}_{3}$ takes place at $1150-1200^{\circ} \mathrm{C}$ in the presence of a $\mathrm{Bi}$-rich liquid phase which forms at $700-800^{\circ} \mathrm{C}$, depending on the exact material composition. Uncontrolled bismuth oxide volatilisation can modify the electrical response of the material since bismuth is critical for the development of the electrically active grain boundaries. On the other hand, material oxygenation is also essential for the electrical behaviour of the material. Consequently the area-volume ratio of the green compacts becomes a relevant parameter for the sintering process. In the present work, the microstructure and current-voltage characteristic of sintered varistors with different area-volume ratio and identical composition have been studied. Depending on the sintering temperature, there is value for the area-volume ratio above which a strong decrease of the electric properties of the varistor device is observed.

Key words: Ceramic varistor, area-volume ratio, sintering, electrical properties.

\section{INTRODUCCIÓN}

Los varistores cerámicos basados en el sistema $\mathrm{ZnO}-\mathrm{Bi}_{2} \mathrm{O}_{3}-$ $\mathrm{Sb}_{2} \mathrm{O}_{3}$ presentan una respuesta intensidad-voltaje no lineal característica, que es consecuencia directa de su microestructura(1-3). El comportamiento no óhmico se origina en los bordes de grano eléctricamente activos, formados por una fase intergranular rica en $\mathrm{Bi}_{2} \mathrm{O}_{3}$ que separa los granos de $\mathrm{ZnO}$ semiconductores $(4,5)$. El varistor cerámico está constituido además por pequeñas cantidades de óxidos dopantes; $\mathrm{Bi}_{2} \mathrm{O}_{3}$, $\mathrm{Sb}_{2} \mathrm{O}_{3}, \mathrm{CoO}, \mathrm{MnO}_{2}$ y $\mathrm{Cr}_{2} \mathrm{O}_{3}$ entre otros, implicados en la mejora de las características eléctricas del varistor. Sin embargo, las reacciones básicas que conforman la microestructura final y que tienen lugar durante la sinterización son las siguientes:

$$
\begin{aligned}
& 4 \mathrm{ZnO}+3 \mathrm{Bi}_{2} \mathrm{O}_{3}+\mathrm{Sb}_{2} \mathrm{O}_{3}+\mathrm{O}_{2} \rightarrow 3 \mathrm{Bi}_{2}\left(\mathrm{Zn}_{4 / 3} \mathrm{Sb}_{2 / 3}\right) \mathrm{O}_{6} \\
& \mathrm{Bi}_{2}\left(\mathrm{Zn}_{4 / 3} \mathrm{Sb}_{2 / 3}\right) \mathrm{O}_{6}+3 \mathrm{ZnO} \rightarrow \mathrm{Zn}_{7} \mathrm{Sb}_{2} \mathrm{O}_{12}+\mathrm{Bi}_{2} \mathrm{O}_{3}(\mathrm{liq})
\end{aligned}
$$

En las primeras etapas de la sinterización aparece una fase intermedia con estructura tipo pirocloro, $\mathrm{Bi}_{2}\left(\mathrm{Zn}_{4 / 3} \mathrm{Sb}_{2 / 3}\right) \mathrm{O}_{6}$, que a temperaturas mas elevadas da lugar a la fase de estructura espinela $\mathrm{Zn}_{7} \mathrm{Sb}_{2} \mathrm{O}_{12^{\prime}}$ (implicada en el control del crecimiento del tamaño de los granos de $\mathrm{ZnO}$ ) y a una fase liquida rica en
$\mathrm{Bi}_{2} \mathrm{O}_{3}$ que tras el enfriamiento se localiza en los huecos creados en el empaquetamiento de granos de $\mathrm{ZnO}$ (1-6). En el procesamiento del varistor son importantes tanto la cantidad de bismuto presente en la composición final, como una adecuada oxigenación del material para el establecimiento de las uniones activas. Durante las primeras etapas de la sinterización, la entrada de $\mathrm{O}_{2}$ en el material permite la oxidación del $\mathrm{Sb}$ y la posterior formación de la fase espinela. Posteriormente, a las temperaturas a las que tiene lugar la sinterización, se produce una volatilización incontrolada de bismuto (7) que junto con una insuficiente oxigenación puede degradar las propiedades eléctricas del material varistor. Estos dos procesos son función de la superficie de contacto con la atmósfera. Un aumento de la superficie produce una mejor oxigenación pero también puede dar lugar a una mayor volatilización de bismuto. La relación área-volumen óptima debe ser aquella que equilibre ambos efectos. En el presente trabajo se estudia el papel que juega una determinada relación área-volumen en la obtención de las propiedades eléctricas de varistores cerámicos, en función de la temperatura de sinterización empleada. 


\section{PROCEDIMIENTO EXPERIMENTAL}

La composición del material empleada en este trabajo es una composición típica de un material varistor reflejada ampliamente en la literatura (6-9): $97.2 \%$ molar de $\mathrm{ZnO}, 0.5 \%$ $\mathrm{Bi}_{2} \mathrm{O}_{3}, 1 \% \mathrm{Sb}_{2} \mathrm{O}_{3}, 0.5 \% \mathrm{MnO}_{2}, 0.5 \% \mathrm{Cr}_{2} \mathrm{O}_{3}$ y $0.3 \%$ de CoO. Las materias primas empleadas son de una pureza superior al 99.99\%. La homogeneización de la mezcla de óxidos se llevó a cabo en medio etanólico mediante agitación. El polvo obtenido se secó en estufa a $60^{\circ} \mathrm{C}$ y se tamizó por debajo de $100 \mu \mathrm{m}$. Las muestras fueron conformadas en forma de cilindros con un diámetro constante de $15 \mathrm{~mm}$ y una altura variable, en función de la masa de material empleada, mediante prensado uniaxial a una presión de $800 \mathrm{Kg} / \mathrm{cm}^{2}$. La sinterización del material se realizó a las temperaturas de 1160 y $1200^{\circ} \mathrm{C}$, durante 2 horas.

La densidad de los cilindros prensados y sinterizados se determinó por el método de Arquímedes obteniéndose en todos los casos valores de densidad de $5.62 \pm 0.02 \mathrm{gr} / \mathrm{cm}^{3}$. La microestructura de los materiales se observó mediante Microscopía Electrónica de Barrido (MEB) en un microscopio Zeiss DSM 950 sobre muestras pulidas y atacadas químicamente con una disolución de $\mathrm{HCl}$ al 5\%. El tamaño de grano promedio se determinó mediante el método intercepción tomando al menos 300 granos. La caracterización eléctrica se llevó a cabo sobre discos cortados con un espesor de $1.5 \mathrm{~mm}$ y electrodados con plata. La respuesta I-V en corriente continua se midió con un multímetro Keithley 2410 provisto de una fuente de alto voltaje de 1100 voltios.

A partir de las curvas I-V se deducen los siguientes parámetros que permiten comparar la bondad del comportamiento varistor: $\mathrm{E}_{\text {eff }}$ es el campo de conmutación para $5 \mathrm{~mA} / \mathrm{cm}^{2}, \mathrm{~J}_{\mathrm{f}}$ es la densidad de corriente de fuga medida para un campo del $85 \%$ del campo de conmutación y el coeficiente de no-linealidad $\alpha$ calculado para valores de densidad de corriente entre 5 y $20 \mathrm{~mA} / \mathrm{cm}^{2}$ a partir de la relación $\mathrm{V} \equiv \mathrm{KI}^{\alpha}$.

\section{RESULTADOS Y DISCUSIÓN}

En la tabla I se muestran los valores de la masa, altura y relación área-volumen que caracterizan a las distintas muestras conformadas en verde, presentando todas ellas una densidad en verde de $3.1 \pm 0.05 \mathrm{gr} / \mathrm{cm}^{3}$. El análisis microestructural de los materiales sinterizados no reveló diferencias en cuanto a las fases cristalinas presentes. El tamaño de grano promedio medido depende directamente de la temperatura de sinterización y no presenta diferencias respecto a la relación área-volumen de la muestra; como se observa en las micrografías de la figura 1 (realizadas sobre los materiales denominados $\mathrm{C}$ y E), el tamaño de grano promedio es $8 \pm 0.5$ $\mathrm{mm}$ para las muestras sinterizadas a $1160^{\circ} \mathrm{C}$ y $10 \pm 0.5 \mathrm{~mm}$ para las sinterizadas a $1200^{\circ} \mathrm{C}$.

TABLA I ESTIMACIÓN DE LA RELACIÓN ÁREA-VOLUMEN PARA MUESTRAS EN VERDE.

\begin{tabular}{|c|c|c|c|c|c|}
\hline & Muestra A & Muestra B & Muestra C & Muestra D & Muestra E \\
\hline Altura (cm) & 0.33 & 0.67 & 1.03 & 1.38 & 1.72 \\
\hline Masa (gr) & 2.0 & 4.0 & 6.0 & 8.0 & 10.0 \\
\hline $\begin{array}{c}\text { Relación } \\
\text { Area/Volumen } \\
\left(\mathrm{cm}^{-1}\right)\end{array}$ & 8.67 & 5.64 & 4.60 & 4.11 & 3.83 \\
\hline
\end{tabular}
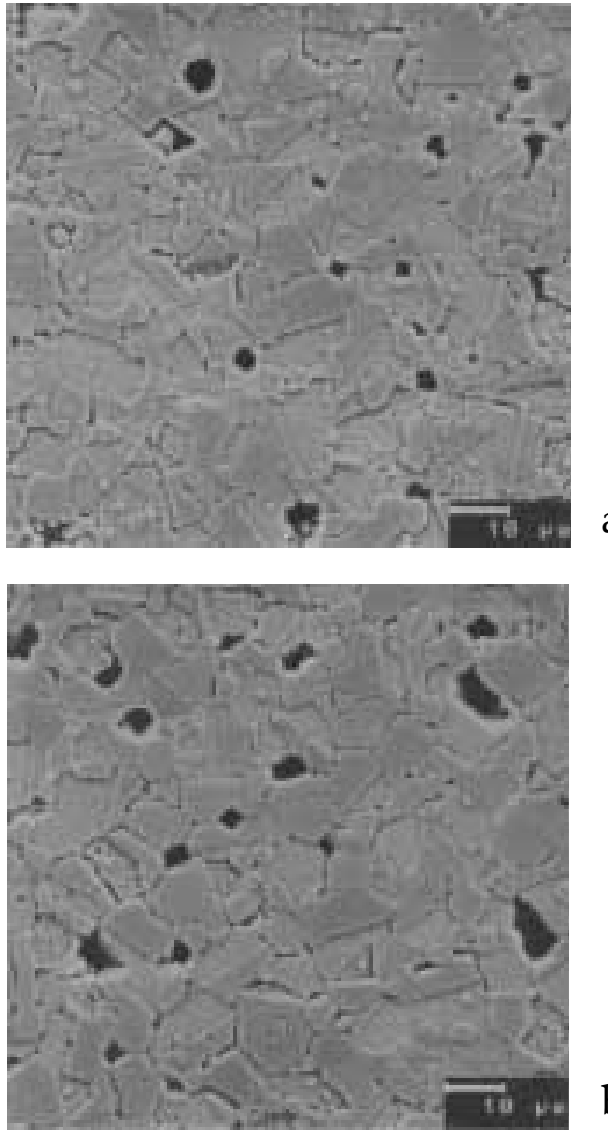

$\mathrm{b}$
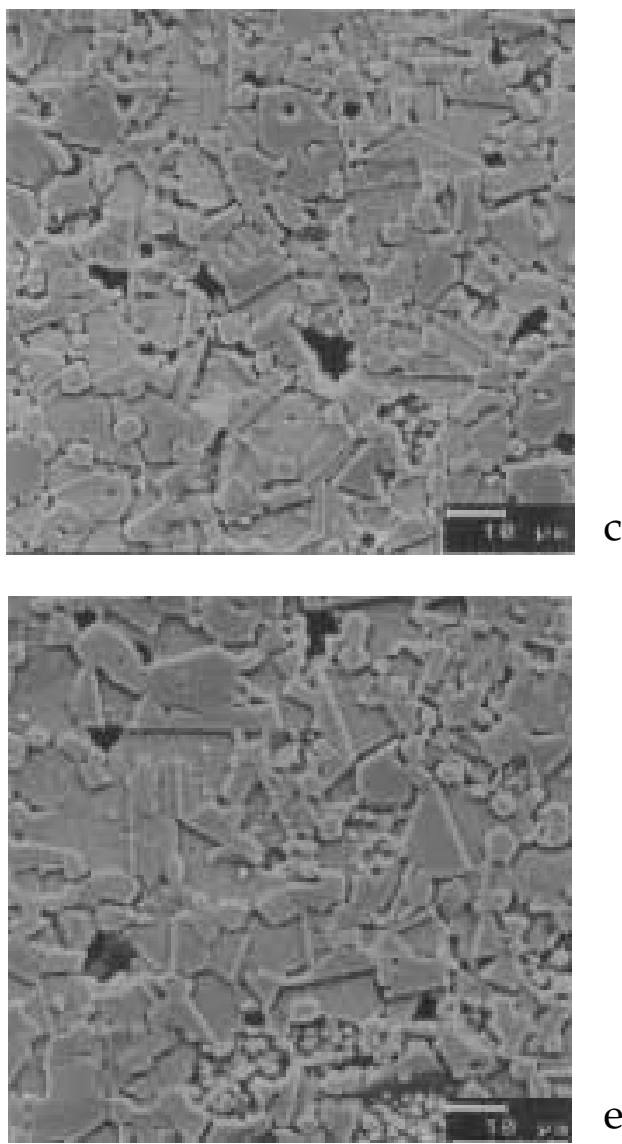

Figura 1. Micrografías obtenidas mediante MEB sobre muestras sinterizadas: A) Muestra $\mathrm{C}$ a $1160^{\circ} \mathrm{C}$. B) Muestra $\mathrm{C}$ a $1200^{\circ} \mathrm{C}$. C) Muestra E a $1160^{\circ} \mathrm{C}$. D) Muestra E a $1200^{\circ} \mathrm{C}$. 


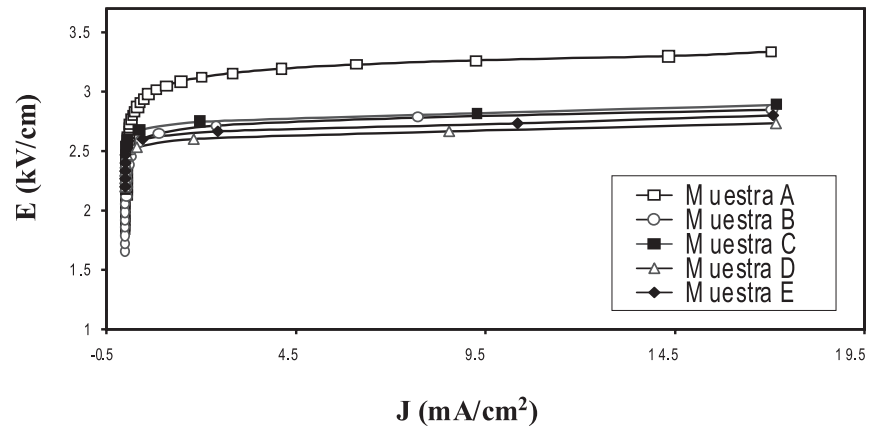

Figura 2. Respuesta Intensidad-Voltaje para los materiales sinterizados a $1160^{\circ} \mathrm{C}$.

TABLA II RELACIÓN AREA/VOLUMEN FRENTE A RESPUESTA ELÉCTRICA PARA LAS MUESTRAS SINTERIZADAS A $1160^{\circ} \mathrm{C}$.

\begin{tabular}{|c|c|c|c|c|}
\hline $\mathbf{1 1 6 0} \mathbf{}^{\mathbf{C}}$ & $\begin{array}{c}\text { Relación } \\
\text { Area/Volumen } \\
\left(\mathbf{c m}^{-1}\right)\end{array}$ & $\mathbf{E}_{\text {eff }} \mathbf{( k V / c m )}$ & $\mathbf{J}_{\mathbf{f}}\left(\mathbf{m A} \mathbf{A} \mathbf{c m}^{\mathbf{2}}\right)$ & $\alpha$ \\
\hline Muestra A & 8.67 & 3.21 & 0.186 & 37 \\
\hline Muestra B & 5.64 & 2.76 & 0.052 & 50 \\
\hline Muestra C & 4.60 & 2.79 & 0.003 & 54 \\
\hline Muestra D & 4.11 & 2.64 & 0.001 & 52 \\
\hline Muestra E & 3.83 & 2.70 & 0.002 & 57 \\
\hline
\end{tabular}

En las figuras 2 y 3 se representa la respuesta I-V de las distintas muestras sinterizadas a las temperaturas de 1160 y $1200^{\circ} \mathrm{C}$ respectivamente. Los parámetros eléctricos que se deducen a partir de estas curvas se resumen en las tablas II y III.

Para las muestras sinterizadas a $1160^{\circ} \mathrm{C}$ con relaciones $\mathrm{A} / \mathrm{V}$ inferiores a $5.64 \mathrm{~cm}^{-1}$ la respuesta eléctrica es muy similar. El campo de conmutación es más elevado que para las sinterizadas a $1200^{\circ} \mathrm{C}$, lo que se corresponde con el menor tamaño de grano. En el caso de la muestra con mayor relación área-volumen sinterizada a $1160^{\circ} \mathrm{C}$, se observa claramente un comportamiento diferente caracterizado por un coeficiente de no-linealidad inferior, una mayor corriente de fugas y un campo de conmutación muy superior al del resto de muestras. Este comportamiento apunta un origen doble, por un lado la disminución del coeficiente de no-linealidad y el aumento de la corriente de fugas indican un descenso del potencial barrera en borde de grano y por otro el aumento del voltaje conmutación parece reflejar una mayor proporción de uniones activas $(1,5)$.

Para las muestras sinterizadas a $1200^{\circ} \mathrm{C}$ se observa una mayor dispersión en la respuesta intensidad-voltaje, si bien de manera análoga al caso anterior, la muestra con mayor relación área-volumen presenta un comportamiento claramente diferenciado. Tanto el coeficiente de no-linealidad como el campo de conmutación son muy inferiores al resto y la corriente de fugas muy superior. En este caso, el origen del comportamiento apunta a un descenso dramático de la altura de la barrera de potencial generada en los bordes de grano eléctricamente activos.

Puesto que las muestras con mayor relación área-volumen

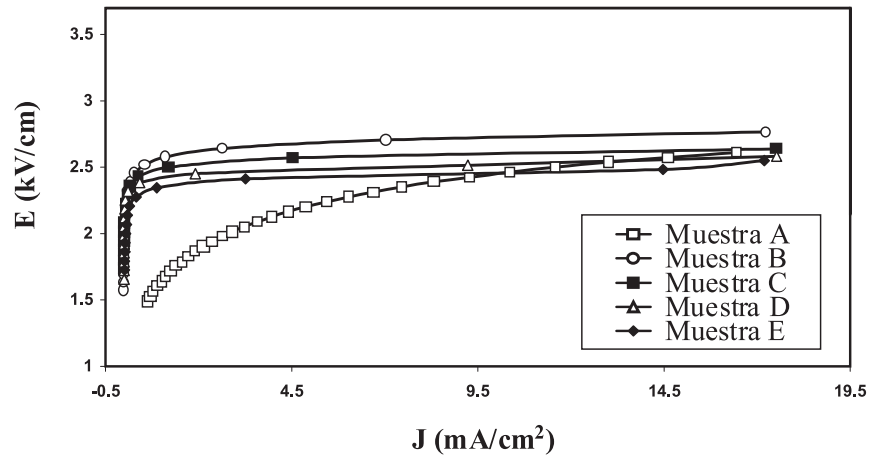

Figura 3. Respuesta Intensidad-Voltaje para los materiales sinterizados a $1200^{\circ} \mathrm{C}$.

TABla III. RELACIÓN AREA/VOLUMEN FRENTE A RESPUESTA ELÉCTRICA PARA LAS MUESTRASSINTERIZADAS A $1200^{\circ} \mathrm{C}$

\begin{tabular}{|c|c|c|c|c|}
\hline $\mathbf{1 2 0 0}{ }^{\mathbf{}} \mathbf{C}$ & $\begin{array}{c}\text { Relación } \\
\text { Area/Volumen } \\
\left(\mathbf{c m}^{-\mathbf{1}}\right)\end{array}$ & $\mathbf{E}_{\text {eff }}(\mathbf{k V} / \mathbf{c m})$ & $\mathbf{J}_{\mathbf{f}}\left(\mathbf{m A} \mathbf{\mathbf { c m } ^ { 2 }}\right)$ & \\
\hline Muestra A & 8.67 & 2.20 & 1.92 & 7 \\
\hline Muestra B & 5.64 & 2.68 & 0.064 & 44 \\
\hline Muestra C & 4.60 & 2.57 & 0.025 & 53 \\
\hline Muestra D & 4.11 & 2.48 & 0.007 & 57 \\
\hline Muestra E & 3.83 & 2.43 & 0.040 & 52 \\
\hline
\end{tabular}

son las que tienen las condiciones más favorables para el proceso de oxigenación del material, su peor comportamiento parece originarse en la volatilización del bismuto. Lógicamente la cinética del proceso es más rápida a una temperatura mayor, por lo que la degradación de las propiedades es mucho más marcada a medida que la temperatura de sinterización es más elevada, llegando a inutilizar el material desde el punto de vista de su aplicación como varistor.

\section{CONCLUSIONES}

La relación área-volumen de los compactos en verde es un parámetro crítico en la obtención de varistores cerámicos basados en el sistema $\mathrm{ZnO}-\mathrm{Bi}_{2} \mathrm{O}_{3}$. La configuración de la barrera de potencial en borde de grano presenta un fuerte dependencia con el contenido en $\mathrm{Bi}^{3+}$ y oxígeno. La volatilización parcial del bismuto limita la máxima relación área-volumen que permite obtener un comportamiento varistor óptimo. Para temperaturas de sinterización comprendidas entre 1160 y $1200^{\circ} \mathrm{C}$ una relación área-volumen en el entorno de 8 $\mathrm{cm}^{-1}$ puede deteriorar la respuesta no-lineal del material invalidando su aplicación como varistor.

\section{AGRADECIMIENTOS}

Este trabajo ha sido realizado en el marco del proyecto C00 1999 AX012 (CICYT). Los autores agradecen a INAEL S.A., KERABEN S.A., IKV S.A., IQB S.A., y al CSIC el apoyo prestado en la realización del trabajo. 


\section{BIBLIOGRAFÍA}

1. D.R. Clarke. "Varistor Ceramics". J. Am. Ceram. Soc. 82 [3] 485-502 (1999)

2. M. Matsuoka. "Nonohmic Properties of Zinc Oxide Ceramics". Jpn. J. Appl. Phys.10 [5] 736-746 (1971).

2. T. K. Gupta. "Application of Zinc Oxide Varistors". J. Am. Ceram. Soc. 73 [7] 1817-1840 (1990).

3. M. Elfwing, R. Österlund, E. Olsson. "Differences in Wetting Characteristics Of $\mathrm{Bi}_{2} \mathrm{O}_{3}$ Polymorphs in $\mathrm{ZnO}$ Varistors Materials". J. Am. Ceram. Soc. 83 [9] 2311-14 (2000).

4. E. Olsson, G. Dunlop, R. Österlund. "Development of Funcional Microstructure during Sintering of a ZnO Varistor Material". J. Am. Ceram. Soc. 76 [1] 65-71 (1993).
5. L. Wu, C-Y. Chen, Y-F. Wei, M-H. Chen, K-C Huang. " The Microstructure of $\mathrm{ZnO}$ varistor Doped with Antimony Oxide". Jpn. J. Appl. Phys. 50 [11-A] 2850-2856 (1991)

6. A.C. Caballero, F.J. Valle, J. A. Martín Rubí. "Determination of dopants in $\mathrm{ZnO}$-based ceramic varistors by $\mathrm{x}$-ray fluorescence and inductively copuled plasma spectrometry". X-Ray Spectrom. 30, 273-279, (2001).

7. M. Inada. "Microstructure of Nonohmic Zinc Oxide Ceramics". Jpn. J. Appl. Phys. 17 [4] 673-677 (1978).

8. A. C. Costa, E. Guedes da Costa, L. N. Santana, L.R Pontes. "Electrical Characteristis of $\mathrm{Ni}_{2} \mathrm{O}_{3}$-doped $\mathrm{ZnO} \cdot \mathrm{Bi}_{2} \mathrm{O}_{3} \cdot \mathrm{CO}_{2} \mathrm{O}_{3} \cdot \mathrm{MnO}_{2}$ varistor systems". Ceramica 44 (287-288) 130-135 (1998).

9. K.O. Magnusson. S. Wiklund. "Interface formation of Bi on ceramic ZnO: A simple model varistor grain boundary". J. Appl. Phys. 76 [11] 7405-7409 (1994)

Recibido: 31.05.01

Aceptado: 10.12 .01

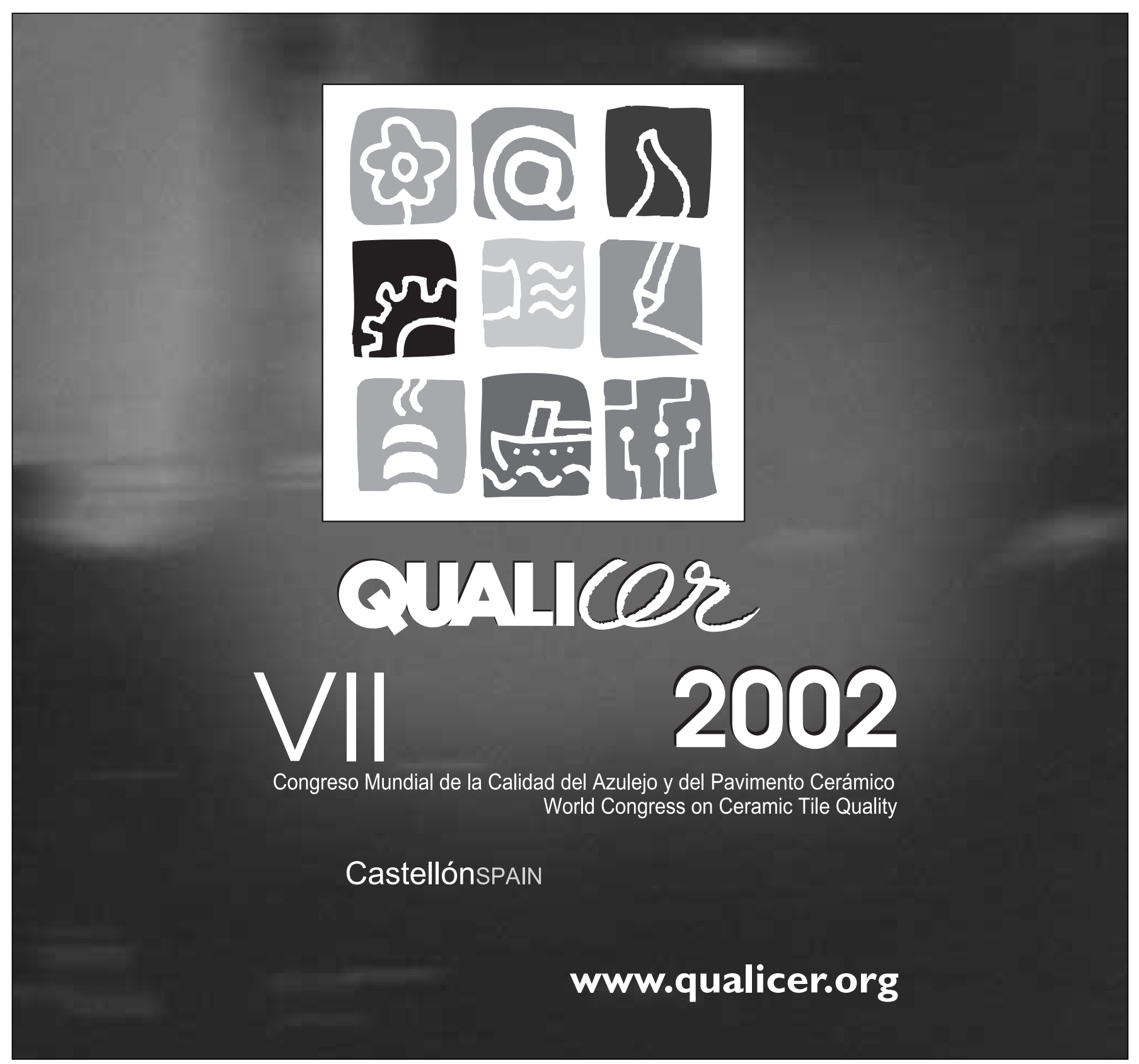

